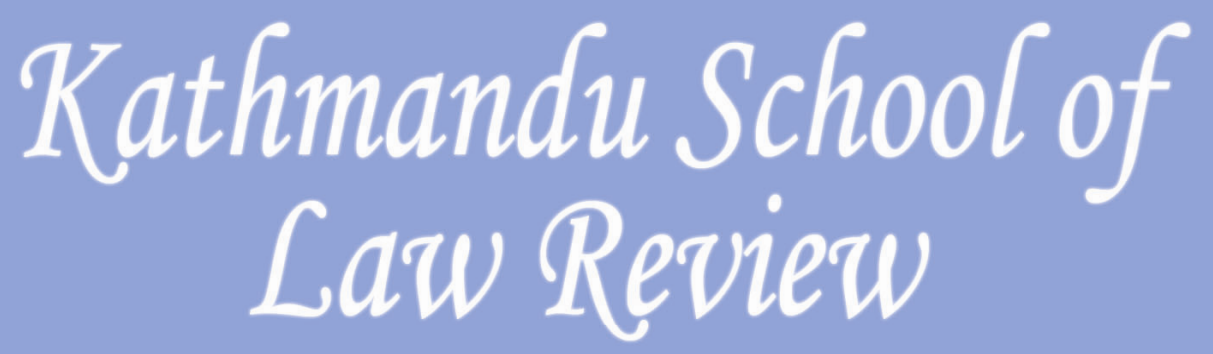

ISSN 2091-2110

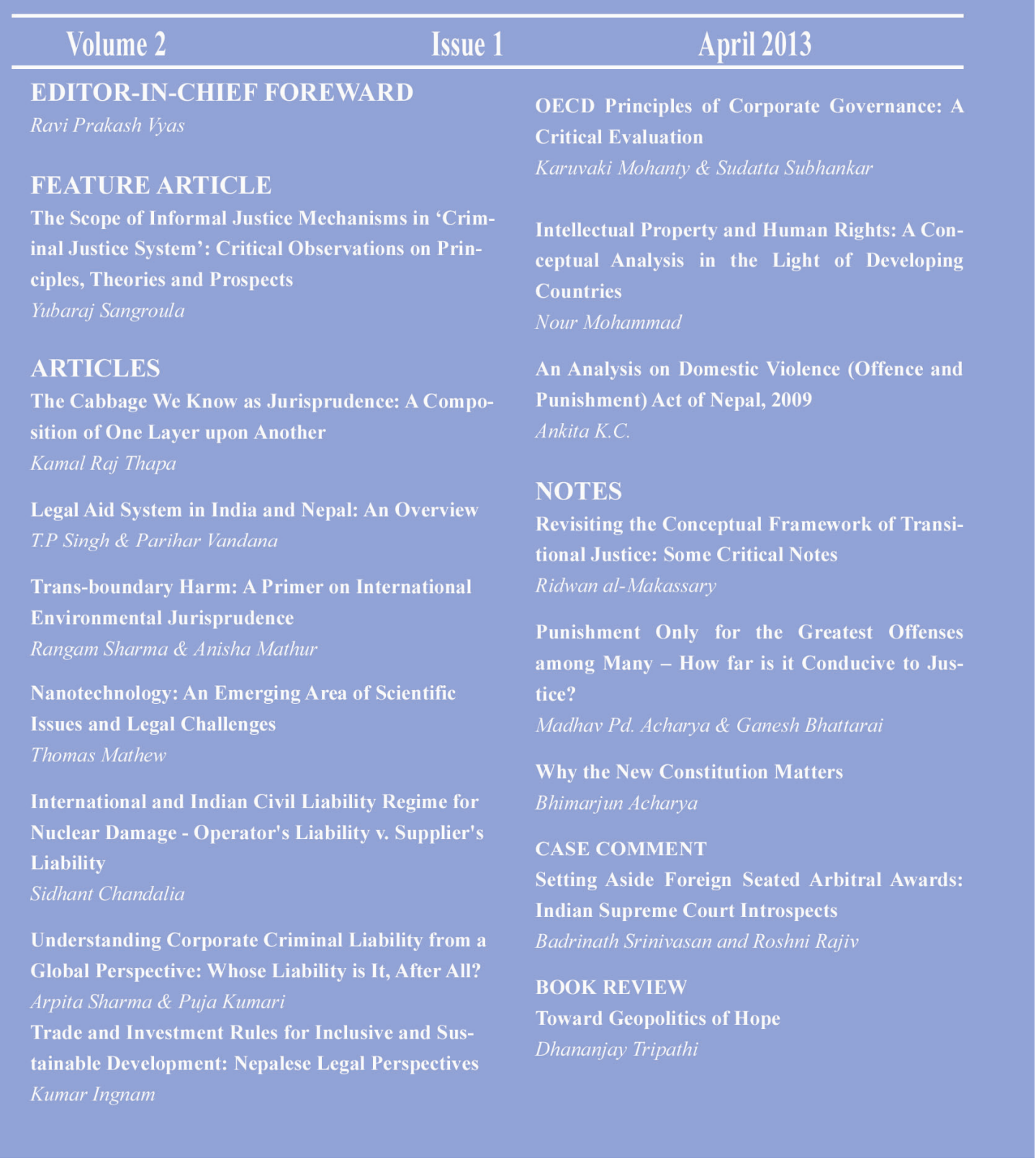




\title{
Nanotechnology: An Emerging Area of Scientific Issues and Legal Challenges
}

\author{
Thomas Mathew ${ }^{1}$
}

\begin{abstract}
Nanotechnology is based on the convergence of several disciplines of science and technology. India though entering late in this field has been able to make a mark, however, there is a long way to go both in the scientific and legal arena. This paper initially focuses on the scientific development of nanotechnology in India and thereafter onvarious acts and regulations that deal with nanotechnology in India.
\end{abstract}

Understanding the 'nano' phenomenon and the investigation of the properties of various nanomaterials, control and maneuvering of matter at the nano scale is generally dealt by nanoscience, whereas nanotechnology involves using tools and methods for the synthesis, analysis, manufacture and application of materials, products and systems that are at the nanometer scale or incorporate facets of the same dimensions ${ }^{2}$. Nanotechnology is based on the convergence of several disciplines of science and technology. The term 'nanotechnology' is by and large used as a reference for both nanoscience and nanotechnology especially in the public domain. In a regulatory context, the definition of nanotechnology can make a significant difference in what is regulated, how it is regulate and how well a regulatory program works. There are a variety of definitions of nanotechnology in circulation such as by the US National Nanotechnology Initiative (NNI) and by the Europeans. ${ }^{3}$

Post liberalization India has embraced the use of emerging technologies for rapid industrialization which has resulted in the current growth rate and significant achievements in domain of science and technology. ${ }^{4}$ The Ministry of Science and Technology of India

\footnotetext{
1 Assistant Professor of Science and Technology, Gujarat National Law University, Gandhinagar, Gujarat, India.

2 The Royal Society \& The Royal Academy of Engineering, 'Report on Nanoscience and nanotechnologies: opportunities and uncertainties' (2004) <http://www.nanotec.org.uk/finalReport.htm> accessed on 28 April 2013.

3 Ibid.

$4 \quad$ D Schaffer, 'India Rising' (2011) 22 TWAS Newsletter Academy of Sciences for the Developing World $10,21$.
} 
administers its functions through three departments: department of science and technology (DST), department of biotechnology (DBT) and department of scientific and industrial research (DSIR). Amongst these, DST is the important one with the objective of promoting new areas of science and technology and to play the role of a nodal department for organizing, coordinating and promoting S\&T activities in India. ${ }^{5}$ In 1997, as an earliest nanotechnology initiative in the policy-making arena in India, a committee was created by DST under Prof. D Nagchoudhary for looking into the prospects of this technology and fund research for 3 years ${ }^{6}$. India, later notwithstanding resource constraints, embarked on a Nano Science and Technology Initiative (NSTI) in 2001, the culmination of which witnessed the initiation of a larger mission mode program-Nanoscience and Technology Mission (Nano mission) in the year $2007^{7}$ with the DST acting as the nodal agency. Nano Mission intended to create the necessary innovation climate for nanotechnology by strengthening basic research through funding support, creating centers of excellence, establishing a chain of shared facilities across the country for optimal use of expensive and sophisticated equipment, fund application oriented R\&D projects, foster public-private partnerships, organize international collaboration and provide effective education and training to researchers and professionals. Apart from DST, some of the main departments and industrial associations involved in the promotion of nanotechnology in India are DBT, DSIR, Department of Information Technology (DIT), Defence Research and Development Organization (DRDO), Department of Industrial Policy and Promotion (DIPP), Confederation of Indian Industries (CII), Associated Chambers of Commerce and Industry of India (ASSOCHAM), Federation of Indian Industry (FICCI), National Institute of Pharmaceutical Education and Research (NIPER), Indian National Science Academy (INSA), Council for Scientific and Industrial Research (CSIR) and Indian Council of Medical Research (ICMR). Thus, there are several regulatory bodies, research bodies, industry organizations and implementing agencies that together create and manage the regulatory landscape for any technology or its application. All the institutions, irrespective of their role and functions, influence regulation directly or indirectly. For instance, even though DST has a clear mandate of promoting nanotechnology research, application and commercialisation, its actions certainly impact the manner in which the overall governance framework is designed and implemented.

The international forums/institutions like Intergovernmental Forum on Chemical Safety (IFCS), International Standards Organization (ISO), and the International Risk Governance

$5 \quad$ Shilpanjali Deshpande Sharma, 'How Resilient is India to Nanotechnology Risks? Examining Current Developments, Capacities and an Approach for Effective Risk Governance and Regulation' (2011) 2(3) European Journal of Law and Technology 1.

6 SERC, Annual Report (2001) 200.

7 Vivek Srivastava, 'Workshop on Nanotechnology: Current status and Challenges' (Indian Institute of Technology Delhi, 2007) < http://www.nanotech-now.com/columns/?article=083, 2007> accessed 1 May 2013. 
Council (IRGC) are characteristically sub-political in nature and as much as they lack effective legitimacy and formal rule making power, they do play a role.

Nanotechnology, whilst promising socio-economic and development gains, also presents potential challenges for environmental and human safety. ${ }^{8}$ Emergence of nanoproducts may also lead to public concerns on aspects like benefits, risks, equity and ethics like the public dispute and debate in the case of Genetically Modified (GM) technologies. There is a growing evidence that the manufacture, use and disposal of nanoproducts manufactured from some nanomaterials that possess unusual physicochemical properties might present potential risks or adverse responses, over conventional chemicals as much as they do have benefits. $^{9}$ Another concern is that the existing risk assessment, management or regulatory frameworks may not be adequate to address nanotechnology risks as are tailored for conventional chemicals. ${ }^{10}$ Hansen has asserted that diversity of nanomaterials serious information deficits reign on the nature of risks, problems with materials characterization and standardization and the knowledge gaps on hazards, exposures and appropriate regulatory thresholds. The traditional ways of evaluating and regulating risks would need to be reconsidered in the case of nanotechnologies; hence, the effective persuasion remains a key question.

In India nanotechnology is positioned as an essential tool in the national development agenda in sectors such as energy, water and health like in most of the developing countries. ${ }^{11}$ Despite a decade long involvement, state remains the main driver for nanotechnology R\&D in India and continues to have a strong hold on the direction of its trajectory. Hence, there is also a thrust to enhance technology development by means of public private partnerships, establishment of incubators etc. Besides initiatives at the centre, state-led initiatives to promote nanotechnology are emerging and contributing to the significant expansion of this technology.

The regulatory interventions in any given technology are required at five main stages of its development and application: i) Research and Development (R\&D) and intellectual property rights (IPR); ii) Production and marketing; iii) Occupational health and safety; iv) Environmental risk management and v) Waste disposal. Despite DST being the nodal department to promote science and technology in India, its mandate does not include risk governance. DST has recently appointed a task force to advice Nano Mission Council to develop a regulatory body for nanotechnology in India. Currently a nanotechnology specific regulation does not exist in India. It would be incorrect to say that nanotechnology

The Royal Society \& The Royal Academy of Engineering (n 2).

A. D Maynard, 'Nanotechnology: Overview and Issues' in Simeonova et al (eds), NanotechnologyToxicological Issues and Environmental Safety (Springer Publications 2007) 1-14.

10 O Renn \& M Roco, White Paper on Nanotechnology Risk Governance (International Risk Governance Council 2007).

11 F Salamanca-Buentelloand et al, 'Nanotechnology and the Developing World' (2005) 2(5) PLoS Med 97. 
and nanoparticles cannot be regulated in the absence of such a dedicated regulation. The source of (legal) regulation is the state, ${ }^{12}$ whereas governance is a much broader term encompassing a gamut of private and public actions that are aimed towards a specific aim. ${ }^{13}$ Thus regulatory framework for a technology will refer to a number of aspects of the production and application of that technology. ${ }^{14}$ Thus, although the regulatory toolkit for technologies are broad and may span a number of sectors seemingly delinked from one another (like intellectual property rights and health for instance) they also reflect the sectoral dynamics that will influence the scope of regulatory action and the choice of regulatory tool selected by regulators. ${ }^{15}$ The various acts and regulations dealing with nanotechnology in India with the above mentioned stages are:

The Patents (Amendment) Act 2005 defines an invention as 'a new product or process involving an inventive step and capable of industrial application' in its section $2(1)(\mathrm{j})$ and also further reinforces this by providing a bar to patentability of 'discoveries' in section 3 (d). The section 3(d) serves two functions: first, it is designed to discourage patent 'evergreening' by prohibiting the grant of a patent on a derivative form of a known substance unless the derivative form has significantly enhanced efficacy. Secondly, it prohibits 'new use patents' by expressly providing that mere discovery of any new property or new use for a known substance is not patentable. The Madras High Court in the Novartis Case $^{16}$ discussed at length and interpreting section 3(d) also chose to take a tough stand on the issue of efficacy. Apart from post-grant opposition clause as per which a patent may be opposed post-grant, the act has quite an elaborate framework for compulsory licensing, which ensures that the public good is served, that the abuse of patent as a monopoly is prevented in order to make way for commercial exploitation of invention by an interested person. According to section 84 of the Act, a compulsory license could be granted on a patent after three years, from the date of grant of that patent. The Act also has an experimental use exemption, which may prove to be an important liability shield for universities seeking to engage in NT research, without having to wade through patent thickets in the field.

Drugs and Cosmetics Act 1940 regulates all aspects of drugs and cosmetics pertaining to their import, manufacture, distribution and sale

National Pharmacovigilance Protocol is a post-marketing tool designed to collate data, analyse it and use the inferences to recommend informed regulatory interventions, besides communicating risks to healthcare professionals and the public, in ensuring the safety of pharmaceutical and related health products.

F Salamanca-Buentello et al, 'Nanotechnology and the Developing World' (2005) 2(5) PLoS Med 97.

C Scott, 'Regulatory Innovation and the Online Consumer' (2004) 26 Law and Policy 477, 506.

See R Susskind, The Future of Law (Oxford University Press 1996).

Ibid.

Novartis AG v. Union of India and others (2006) WN 24754. 
The Medical Devices Regulation Bill 2006 though not been enacted till date consolidates laws related to medical devices and establishes a Medical Device Regulatory Authority of India for establishing and maintaining a national system of controls relating to quality, safety, efficacy and availability of medical devices that are used in India.

Insecticides Act 1968 was legislated to regulate the import, manufacture, sale, transport, distribution and use of insecticides with a view to prevent risk to human beings or animals.

Food Safety and Standards Act 2006 lays down science-based standards, and regulates manufacture, storage, distribution, sale and import of food articles to ensure safe and wholesome food. Safety of food and food products were earlier prevented by the Food Adulteration Act.

As put by Breggins and John, generation and disposal of nanowaste is perceived to be a great concern in rapid advancement of nanotechnology and the occupational health and safety (OHS) in India is governed by the following:

Factories Act 1948 was enacted with a view to protect the workers of an establishment, puts a duty on the occupier to make effective arrangements in every factory for the treatment of wastes and effluents due to the manufacturing process to make them innocuous and for their disposal.

Hazardous Material (Management, Handling and Transboundary Movement) Rules 2007 can be made applicable to nanotechnology waste, albeit with certain changes in the text and implementation of these rules.

The Bio-Medical Waste (Management and Handling) Rules 1998 issued under the Environment Protection Act (EPA) enjoins every occupier of an institution generating biomedical with a duty to take all the steps to ensure that any bio-medical waste is handled without any adverse effect to human health and the environment.

The Municipal Solid Wastes (Management and Handling) Rules 2000 identifies municipal bodies as the responsible agencies for infrastructure development for collection, storage, segregation, transportation, processing and disposal of municipal solid wastes.

Environment Protection Act (EPA) is an umbrella legislation under which several notifications and rules have been passed and most environmental initiatives have been taken. The Act in the nature of an enabling legislation is designed in such a manner that there is space to address any of the environmental concerns by way of subordinate legislation or powers and functions of the designated officials. One of the earliest environment specific legislation of India is the Water Pollution Act which takes pollution within its ambit by way of trade effluents and new discharges. The Air Pollution Act, designed on similar lines, empowers the State board to inspect any control equipment, industrial plant or manufacturing process and to give, by order, such directions to such 
persons as it may consider necessary to take steps for the prevention, control or abatement of air pollution.

Public Liability Insurance Act provides for a framework for public liability insurance for the purpose of providing immediate relief to the persons affected by accident occurring while handling any hazardous substance.

The regulatory and standard setting institutions in India apart from DST are:

Central Drug Standard Control Organization (CDSCO) is the primary drug regulatory authority in India. It essentially coordinates all the functions relating to quality control of imported drugs, coordination of activities between the state authorities, new drug approvals for drugs manufactured and imported.

Bureau of Indian Standards (BIS) is the chief Standard Setting body in India and sets voluntary standards to indicate the quality of a product. BIS standards are generally voluntary but some of them become mandatory when notified to that effect by the government.

Environment regulatory agencies are the State Pollution Control Committees, the Central Pollution Control Board and the State Pollution Control Boards.

NIPER is developing regulatory approval guidelines for nanotechnology based drugs and standards for toxicological tests in nano based drug delivery systems. Indian government is planning to set up a regulatory board to examine all new nanotechnology based products. It has formed four national mirror working groups to review the work on: 1) terminology and nomenclature; 2) measurement and characterization; 3) Health, Safety and Environment and 4) Materials specification. Under BIS, development taking place are: National standard on use of Atomic Force Microscope for Characterization and Evaluation of Nanomaterials, Electron Microscopic Characterization of Multi-wall Carbon Nanotubes, Luminescent Nanomaterials and Magnetic Nanoparticles and Standard on Toxicity of Zinc Oxide Nanomaterials

India, though entering late in the field of nanotechnology, has been able to make a mark in this field however there is a long way to go both in the scientific and legal arena. The key challenges that may be faced by the regulatory institutions are: i) regulatory capacity for formulation of rules, policies and guidelines, as well as implementing it; ii) flow of information and iii) lack of capacity which can also be linked to informational asymmetry. A smooth flow of information is necessary for building institutional capacity and taking regulatory measures.

$* * * * * * * * * * * * * * * * * * *$ 